\title{
Substantive antimicrobial activity in chlorhexidine-treated human root dentin
}

\author{
Bettina Basrani, DDS, PhD, ${ }^{a}$ J. Miguel Santos, DDS, ${ }^{\mathrm{b}}$ Leo Tjäderhane, DDS, $\mathrm{PhD},{ }^{\mathrm{c}}$ \\ Helen Grad, MSc, PHM, ${ }^{\mathrm{d}}$ Omer Gorduysus, DDS, PhD, ${ }^{\mathrm{e}}$ Junfu Huang, PhD, ${ }^{\mathrm{f}}$ \\ Herenia P. Lawrence, DDS, MSc, PhD, ${ }^{\mathrm{g}}$ and Shimon Friedman, DMD, ${ }^{\mathrm{h}}$ Toronto, Ontario, \\ Canada; Buenos Aires, Argentina; Coimbra, Portugal; and Ankara, Turkey \\ UNIVERSITY OF TORONTO, MAIMONIDES UNIVERSITY, UNIVERSITY OF COIMBRA, AND HACETTEPE \\ UNIVERSITY
}

\begin{abstract}
Objective. The aim of this in vitro study was to assess the substantive antimicrobial activity of different medicaments in human root dentin.

Study design. Canals of 98 roots were enlarged to standard size and medicated for 7 days with the following: (1) $2 \%$ chlorhexidine (CHX) gel, (2) $0.2 \% \mathrm{CHX}$ gel, (3) $2 \% \mathrm{CHX}$ solution, (4) $\mathrm{Ca}(\mathrm{OH})_{2},(5) \mathrm{Ca}(\mathrm{OH})_{2}+0.2 \% \mathrm{CHX}$ gel, (6) $2 \%$ $\mathrm{CHX}$ solution + a 25\% CHX-containing controlled-release device, (7) saline, and (8) gel vehicle. After medication, canals were inoculated with Enterococcus faecalis for 21 days. Dentin samples were collected with Gates-Glidden burs into brain heart infusion broth, and bacterial growth was assessed with spectrophotometric analysis of optical density after 72 hours of incubation.
\end{abstract}

Results. Mean optical densities were significantly lower for groups with $2 \% \mathrm{CHX}(1,3$, and 6$)$ when compared with those of the controls $(P<.05$, analysis of variance with the Tukey test). Other groups did not differ significantly from the controls.

Conclusions. Canal dressing for 1 week with $2 \% \mathrm{CHX}$ may provide residual antimicrobial activity against $E$ faecalis. (Oral Surg Oral Med Oral Pathol Oral Radiol Endod 2002;94:240-5)

One of the concerns regarding the outcome of endodontic treatment is the potential for root canal reinfection after treatment, even if the canal was thoroughly disinfected before root filling. Reinfection and consequent treatment failure can occur because of regrowth of residual bacteria that survived the treatment procedure. ${ }^{1-3}$ Also, because secondary caries or leakage may

\footnotetext{
${ }^{a}$ Assistant Professor, Department of Endodontics, Faculty of Dentistry, University of Toronto, Ontario, Canada; Department of Endodontics, Faculty of Dentistry, Maimonides University, Buenos Aires, Argentina; and Department of Dental Clinical Sciences, Dalhousie University, Halifax, Nova Scotia, Canada.

${ }^{b}$ Assistant Professor, Department of Dentistry, Faculty of Medicine, University of Coimbra, Portugal.

${ }^{\mathrm{c}}$ Assistant Professor, Department of Endodontics, Faculty of Dentistry, University of Toronto, Ontario, Canada.

${ }^{\mathrm{d}}$ Senior Lecturer, Department of Pharmacology, Faculty of Medicine, Faculty of Dentistry, University of Toronto, Ontario, Canada.

${ }^{\mathrm{e}}$ Assistant Professor, Department of Endodontics, Faculty of Dentistry, Hacettepe University, Ankara, Turkey.

${ }^{\mathrm{f}}$ Assistant Professor, Faculty of Pharmacy, University of Toronto, Ontario, Canada.

${ }^{\mathrm{g}}$ Assistant Professor, Community Dentistry, Faculty of Dentistry, University of Toronto, Ontario, Canada.

${ }^{\mathrm{h}}$ Professor and Head, Department of Endodontics, University of Toronto, Ontario, Canada.

Received for publication Oct 8, 2001; returned for revision Jan 8, 2002; accepted for publication Jan 29, 2002.

(C) 2002 by Mosby, Inc.

$1079-2104 / 2002 / \$ 35.00+0 \quad \mathbf{7 / 1 5 / 1 2 4 0 0 2}$

doi:10.1067/moe.2002.124002
}

allow later ingress of new bacteria into root canals, ${ }^{4}$ it is desirable to develop therapeutic measures that would effectively prevent reinfection and, thus, potentially improve the outcome of endodontic treatment.

One treatment protocol that has been suggested to prevent root canal reinfection in vitro is medication with chlorhexidine gluconate (CHX). ${ }^{5} \mathrm{CHX}$ is a broadspectrum antibacterial agent ${ }^{6,7}$ whose antimicrobial efficacy equals that of the conventional root canal irrigants and medicaments. ${ }^{8-17}$ Unlike the conventional medicaments, the positively charged molecules of CHX can adsorb onto the dentin ${ }^{6}$ and prevent microbial colonization on the dentin surface for some time. ${ }^{18}$ An antimicrobial effect sustained over time is referred to as substantive antimicrobial activity (SAA). ${ }^{19}$ When CHX is used as a root canal irrigant, the antimicrobial effect is short-lived.5,20 For long-term SAA to be achieved, the dentin must be exposed to CHX for a longer time than that afforded by irrigation. ${ }^{21-23}$

A number of studies have demonstrated CHX-affected SAA in bovine root dentin. Komorowski et $\mathrm{al}^{21}$ used $0.2 \%$ CHX solution for 7 days. Heling et $a^{23}$ used a biodegradable controlled-release device (CRD) for the delivery of CHX for 7 days, whereas Lenet et al $^{24}$ used both a nondegradable CRD and a gel vehicle for 7 days. The root canals of bovine teeth are considerably larger than those of human teeth; therefore, the volume of CHX available to interact with the dentin is rela- 
tively large. To better simulate the potential clinical application of CHX, this study sought to assess the efficacy of CHX in different concentrations and modes of applications with respect to providing SAA in the root dentin of human teeth.

\section{MATERIAL AND METHODS \\ Preparation of specimens}

Ninety-eight freshly extracted human teeth were immersed in $0.5 \% \mathrm{NaOCl}$ for 24 hours to remove organic debris and disinfect the surface. The crowns and apices of the teeth were resected with a water-cooled, rotating diamond saw to produce uniform, 6-mm-long specimens. Cementum was removed from the surface of the specimens by using a sanding wheel mounted in a Dremel Multipro Rotary Tool model \#395 (Dremel, Racine, Wis) at $300 \mathrm{rpm}$ to establish an approximate external diameter of $4 \mathrm{~mm}$. The root canal of each specimen was enlarged throughout with a \#3 GatesGlidden drill to standardize its internal diameter. To prevent dehydration, specimens were kept in water during all procedures. Organic and inorganic debris was removed by immersing the specimens in an ultrasonic bath of $17 \%$ EDTA followed by $0.5 \% \mathrm{NaOCl}$, each for 5 minutes. The efficacy of the described procedure in the total removal of the smear layer has been demonstrated elsewhere. ${ }^{21,25}$

The root specimens were placed in test tubes containing brain heart infusion (BHI) broth (Difco Laboratories, Baltimore, Md), autoclaved for 30 minutes at $121^{\circ} \mathrm{C}$, and incubated for 24 hours at $37^{\circ} \mathrm{C}$ to confirm sterility by the absence of turbidity. The sterilized specimens were immersed in an ultrasonic bath of fresh BHI broth for 15 minutes to enhance penetration of the broth into the dentinal tubules. They were blotted dry with sterile paper, coated externally with 2 layers of nail varnish, and mounted in the bottom of a sterile Petri dish with sticky wax.

\section{Medication}

The specimens were randomly divided into 6 experimental groups (1-6) and 2 control groups (7 and 8). Under aseptic conditions, the root canals were medicated with one of the following (Table): group 1, 2\% CHX gel; group 2, 0.2\% CHX gel; group 3, $2 \%$ CHX aqueous solution; group 4 , calcium hydroxide powder mixed with vehicle gel; group 5 , calcium hydroxide mixed with $0.2 \%$ CHX gel; group 6, 2\% CHX solution with a single 25\% CHX-containing CRD; group 7, sterile saline; and group 8 , vehicle gel alone. The prepared canal of each specimen was injected with the intracanal medicament until full $(0.1 \mathrm{~mL})$. The CRD consisted of a core of CHX dissolved in water-permeable polymers, encased in nondegradable polymer film
Table. Description of the groups

\begin{tabular}{crcc}
\hline Group & $n$ & Medication & Vehicle \\
\hline 1 & 15 & $2 \% \mathrm{CHX}$ & CMC gel \\
2 & 15 & $0.2 \% \mathrm{CHX}$ & CMC gel \\
3 & 15 & $2 \% \mathrm{CHX}$ & Water \\
4 & 15 & $\mathrm{Ca}(\mathrm{OH})_{2}$ & CMC gel \\
5 & 15 & $\mathrm{Ca}(\mathrm{OH})_{2}+0.2 \% \mathrm{CHX}$ & $\mathrm{CMC}$ gel \\
6 & 13 & $\mathrm{CRD}+2 \% \mathrm{CHX}$ & $\mathrm{CRD}+$ water \\
7 & 5 & Control & Water \\
8 & 5 & Control & CMC gel \\
\hline
\end{tabular}

CHX, Chlorhexidine gluconate 20\% BP lot \#9915, Wiler Fine Chemicals, London, Ontario, Canada.

$\mathrm{Ca}(\mathrm{OH})_{2}$, Calcium hydroxide USP lot \#196415/8381, BDH Fine Chemicals, Toronto, Ontario, Canada.

$C R D$, An experimental controlled-release device containing $25 \%$ CHX by weight, University of Toronto, Ontario, Canada, with $2 \%$ CHX solution. CMC, Methylcellulose 4000 USP lot \#35933, Wiler Fine Chemicals, London, Ontario, Canada.

coating formed to resemble a gutta-percha cone. Its characteristics have been described in detail elsewhere. ${ }^{26}$

After placement of the medications or control solutions, the specimens were coronally sealed with wax and incubated at $37^{\circ} \mathrm{C}$ for 7 days. Once placed, the medications were not replenished.

\section{Inoculation}

At the end of the medication period, the medications were washed out and the canals were rinsed with $10 \mathrm{~mL}$ of sterile saline and blotted dry with sterile paper points. An inoculum of Enterococcus faecalis (ATCC 29212) grown overnight in BHI broth and adjusted to 0.5 turbidity reading of the McFarland scale $\left(1.5 \times 10^{8}\right.$ bacteria/mL $)^{27}$ was then injected into each canal. The inoculated specimens were incubated at $37^{\circ} \mathrm{C}$ for 21 days. Fresh inoculum was added every other day, and sterile BHI broth was added daily to keep the canals full. Supernatant samples were cultured weekly to confirm the viability and the purity of the inoculum.

\section{Root dentin sampling}

At the end of the inoculation period, each canal was thoroughly rinsed with $10 \mathrm{~mL}$ of sterile saline and blotted dry with sterile paper points. Two samples of root dentin were then obtained by enlarging the canal with sterile Gates-Glidden drills, as follows (Fig 1): the "inner" sample was obtained with drill \#4 to a depth of $0.1 \mathrm{~mm}$ from the root canal surface, and the "outer" sample was then obtained with drill \#5 to a depth of 0.2 $\mathrm{mm}$ from the root canal surface. Each dentin sample was collected into a separate sterile vial containing 3 $\mathrm{mL}$ of sterile fresh BHI broth and was incubated at $37^{\circ} \mathrm{C}$ for 72 hours to allow the growth of any bacteria 


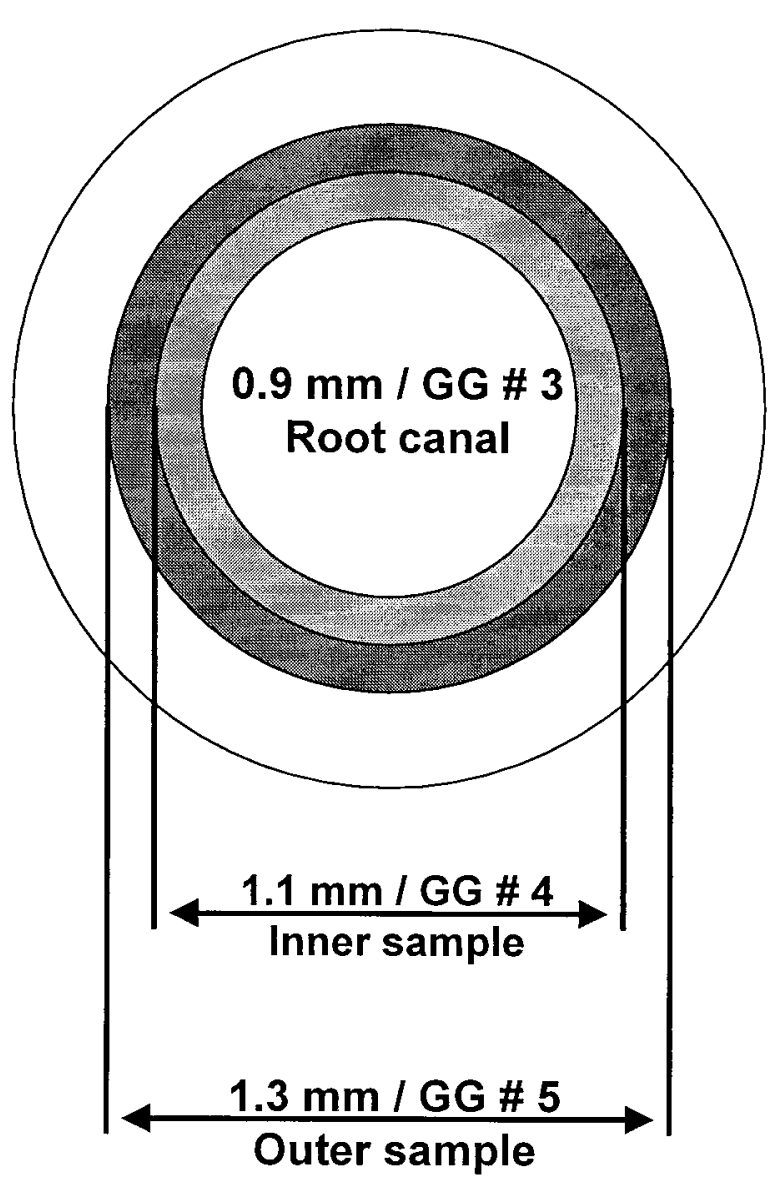

Fig 1. Schematic representation of the circumferential sampling of the dentin. Root canals initially standardized with \#3 Gates-Glidden $(G G)$ drill were enlarged with \#4 (inner sample) and \#5 (outer sample) drills, and the dentin shavings were collected for analysis of bacterial growth.

harbored in the dentin. When the broth became turbid, indicating bacterial growth, it was cultured to ensure the purity of $E$ faecalis.

\section{Data analysis}

After 72 hours, the optical density (OD) of the broth was read by using an Ultrospec III spectrophotometer (Pharmacia LKB Biochrom Ltd, Cambridge, England) at a $600-\mathrm{nm}$ wavelength. OD readings were adjusted to the OD value of fresh, sterile BHI broth as baseline. Within each group, the mean OD values for the inner and outer samples were compared with those of the paired-samples $t$ test. Repeated-measures analysis of variance, taking into account the paired nature of sample collection, was used to analyze the differences among the groups. The Tukey post hoc test was then used to compare individual groups, separately for the inner and outer samples. Statistical tests were 2-tailed and interpreted at the 5\% significance level.

\section{RESULTS}

The OD values observed for the inner and outer samples of all groups are graphically plotted in Fig 2, A and $B$, respectively. In group 3 (2\% CHX solution), the inner samples had significantly lower OD values than the outer samples $(P<.04)$. In group 4 (calcium hydroxide with vehicle gel) the inner samples had significantly higher OD values than the outer samples $(P<.03)$. In all other groups, the OD values of the inner and outer samples did not differ significantly.

Samples from groups 1, 3, and 6 (2\% CHX in gel, solution, and CRD, respectively) had significantly lower OD values than those from groups 7 and 8 (positive controls)Cfor both the inner and the outer samples. Samples from groups 4 and 5 (calcium hydroxide without and with $0.2 \% \mathrm{CHX}$, respectively) did not significantly differ from those from groups 7 and 8 . The outer samples from group $2(0.2 \% \mathrm{CHX}$ gel) had a significantly lower OD value than did those from groups 7 and 8, but this value was not different from all the other groups; however, the inner samples did not differ significantly from all the other groups.

\section{DISCUSSION}

The experimental model used in this study was adapted from that established by Ørstavik and Haapasalo ${ }^{25,28}$ for the study of infection and disinfection of the dentinal tubules. That original model has been modified by several researchers to adapt it to the assessment of antimicrobial activity in root dentin after medication. ${ }^{21-23}$ In the present study, the model was further modified by adapting it to extracted human teeth rather than the previously used bovine incisors. This modification was considered appropriate because of the marked difference in diameter between the canals of bovine and human teeth. The standardized bovine canals in some of the previous studies are more than 3 $\mathrm{mm}$ in diameter, ${ }^{24}$ which is more than 3 -fold that of the standardized canals in this study. Accordingly, the canal volume of the bovine root specimen is almost 10-fold larger than that of the human root specimen of the same length. Because SAA imparted by CHX is known to depend on the amount of CHX molecules available to interact with the dentin, ${ }^{7}$ the "extra large" canals and quantity of CHX in the bovine root specimens may not be relevant to the possible clinical application of CHX in the usual canals of human teeth. Particularly, immersion of a single CRD in a large amount of CHX may misrepresent the clinical application of the CRD as it was intended.

E faecalis was chosen for the inoculum in this study 

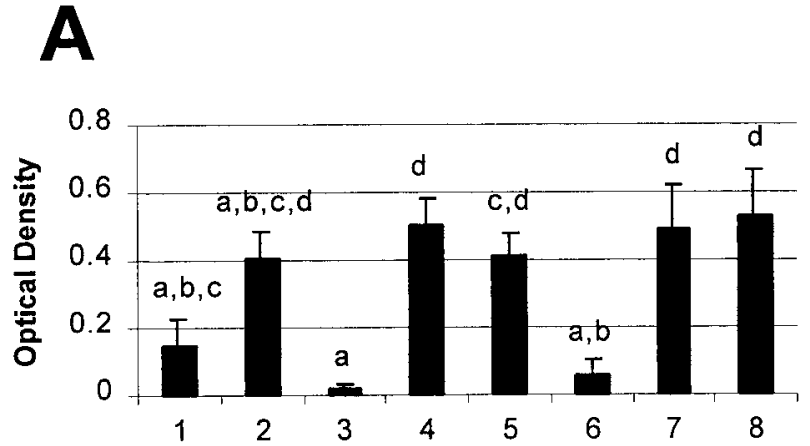

\section{1: $2 \% \mathrm{CHX}$ gel}

2: $0.2 \% \mathrm{CHX}$ gel

3: $2 \% \mathrm{CHX}$ solution

4: $\mathrm{Ca}(\mathrm{OH})_{2}$

5: $\mathrm{Ca}(\mathrm{OH})_{2}+0.2 \% \mathrm{CHX}$ gel

6: $\mathrm{CRD}+2 \% \mathrm{CHX}$ solution

7: Control - gel vehicle

8: Control - water
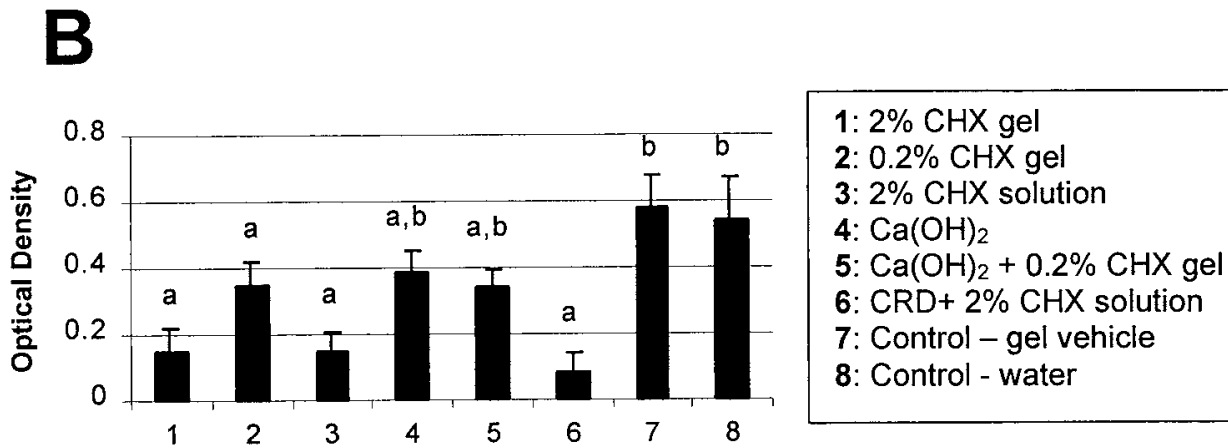

Fig 2. Optical density values (mean $\pm \mathrm{SE}$ ) in broth incubated with dentin samples from human roots treated with test and control intracanal medicaments. The bars marked with the same letter did not show statistically significant differences from each other in the statistical analysis $(P>.05)$. A, Inner samples collected at a depth of $0.1 \mathrm{~mm}$. B, Outer samples collected at a depth of $0.2 \mathrm{~mm}$.

because it is considerably resistant to the common intracanal medication with calcium hydroxide $25,29,30$ and frequently associated with persistent disease after endodontic treatment. ${ }^{3,31-33}$ Because alternative therapeutic modalities that are effective against $E$ faecalis are desirable, ${ }^{9}$ the use of this particular microbe to test potential therapeutic modalities is appropriate. In addition, E faecalis is a nonfastidious microbe that is relatively easy to culture and it has been implemented successfully in most studies by using the original model. ${ }^{21-25}$

A period of 21 days was selected for the assessment of SAA imparted by CHX, as in our previous studies. ${ }^{21,24}$ Clearly, this period can not be considered "long term" where the lifetime of a tooth is concerned; however, it is 3 times longer than that tested by other researchers. ${ }^{22,23,25}$ It does represent an attempt to study SAA over longer periods of time.

The specimens were treated for 1 week with a variety of medications; then, the ability of E faecalis to colonize the root dentin was measured to assess any antimicrobial activity imparted to the root dentin. Of the various experimental and control medications, only those containing 2\% CHX demonstrated resistance to microbial colonization significantly greater than that of the positive controls. This finding confirms the CHXimparted SAA in root dentin documented by other researchers. ${ }^{21,24}$ In fact, it goes further to demonstrate the dependence of SAA on the concentration of CHX, as suggested by Komorowski et al. ${ }^{21}$ Because SAA depends on the amount of CHX molecules available to interact with the dentin, medicating the canal with a more concentrated CHX should result in increased resistance to microbial colonization. The results of this study suggest that, in contrast to the large bovine canals in which $0.2 \%$ CHX effectively imparts SAA, ${ }^{21-23}$ in the small human canals a higher concentration of $\mathrm{CHX}$ was required to impart a comparable effect.

Calcium hydroxide was also tested in the present study because of its prevalent clinical application as intracanal medication. It is known to effectively kill most endodontic pathogens, with the exception of very few, such as E faecalis ${ }^{22,25}$ and Candida albicans. ${ }^{34}$ Calcium hydroxide combined with $0.5 \% \mathrm{CHX}$ has been suggested as an effective killer of $C$ albicans ${ }^{34}$ and a potential substitute for the use of calcium hydroxide alone. ${ }^{34}$ This premise requires systematic investigation, ${ }^{34}$ with SAA being one aspect of it. Because the 
OD values in both calcium hydroxide groups did not differ from those of the controls, the results clearly demonstrate the inability of calcium hydroxide alone or in combination with $0.2 \% \mathrm{CHX}$ to impart SAA into root dentin when it is applied as an intracanal medicament for 1 week. Previous studies have also shown that calcium hydroxide alone does not impart SAA. ${ }^{21-24}$

This study assessed 3 modalities of application of CHX for root canal medication: (1) CHX solution, (2) CHX gel, and (3) CHX-containing CRD immersed in CHX solution. The first modality is the easiest to use clinically, and the results of this study confirm that it did impart SAA when applied for 1 week. However, the initial quantity of CHX solution in the canal is limited, and it is assumed that in clinical application, some of it will be lost by diffusion. Consequently, the amount of CHX molecules available may be critically depleted. Because patients may not return for a subsequent treatment session within 1 week, the intracanal medicament should posses long-term antimicrobial properties to prevent root canal contamination for extended periods. If the CHX solution is depleted, there is a risk that the canal might remain essentially unprotected against microbial contamination.

Two percent CHX gel emerged as an effective intracanal medicament in our previous study ${ }^{24}$ and was again confirmed, in this study, to impart SAA. For an intracanal medicament to be suitable for clinical application, its complete removal from the canal should be easy to ensure effective sealing of the root filling. ${ }^{35}$ For example, there are some concerns regarding the removal of calcium hydroxide paste. ${ }^{35}$ Similarly, the inability to verify total removal of the CHX gel from the canal could be a concern ${ }^{24}$ because further instrumentation before root filling is contraindicated to prevent loss of the CHX-treated dentin surface. According to a recent 1-year in vitro leakage study, ${ }^{36}$ a $\mathrm{CHX}$ gel used to medicate the canals does not appear to interfere with the seal; however, further studies are required to dismiss the concerns regarding gel residue on the canal walls.

A CHX-containing CRD is intended to replenish the CHX in the canal and, thus, to sustain a high level of CHX molecules available for interaction with root dentin. Researchers have developed degradable CRDs for endodontic applications. 22,23,37 Those devices are expected to degrade in the canal over time; however, in clinical application, it may be challenging to verify whether they degraded completely or partially. Therefore, there is a risk that fragments of the CRD might remain in the canal and interfere with root filling. In contrast, the removal of the CRD used in this study was not a concern because of its nondegradable design. In fact, after 1 week the devices remained intact; there- fore, they were simply withdrawn from the canal. For the nondegradable CRD used in this study, the steady release of CHX molecules from the CRD was confirmed over a period of at least 45 days. ${ }^{26}$ In our previous study in bovine teeth, ${ }^{24}$ a single CRD was immersed in a large volume of saline in each canal; this method failed to impart SAA. In contrast, in this study the CRD immersed in 2\% CHX solution did impart SAA as expected.

An advantage of the CRD over the 2\% CHX solution or gel could not be claimed on the basis of this study because the differences among the groups were not significant. However, as suggested earlier, further studies making use of longer application periods of the medicaments and lengthier bacterial inoculation periods may help us determine the efficacy of the different CHX application modalities with respect to imparting SAA. In vivo experiments in animal models, such as that for coronal inoculation established by Friedman et al, ${ }^{4}$ appear to be the next logical step in the assessment of the role of CHX and the different application modalities in imparting clinically meaningful SAA.

The use of OD to measure the results may have had some effect on the outcome of the study because variation in the early log phase of growth may result in some variation in the final readings, especially after the long growth period (72 hours) used in this study. In addition, the possible carryover effect of CHX in the dentin samples may have made an impact on the bacterial growth in the broth. Additional culturing of diluted broth samples for colony-forming units could have resulted in more detailed information. However, the culturing of dilutions for colony-forming units in an experiment with such a large number of samples (eg, the 98 used in this study) would have been a laborious task (requiring at least 196 cultures with only 2 dilutions) and could not be conducted in this study. In addition, the small variation in the OD readings within the groups indicates that the variations between individual samples were not significant. On the other hand, the long growth period before OD analyses most likely reduces the magnitude of the CHX carryover effect on the results. We, therefore, believe that, despite its shortcomings, this study used analytical methods for that are sufficient to validate the conclusions drawn.

In this study, the CHX medicaments were tested in vitro with $E$ faecalis in monoculture. However, endodontic diseases are primarily caused by mixed infections. ${ }^{1}$ The medicament that is effective against a single microbe may not necessarily be effective against a complex microbial flora in vivo. Therefore, further studies are indicated to appraise the efficacy of CHX with respect to imparting SAA against other recognized endodontic pathogens. 
We thank Dr Dennis Cvitkovitch and Kirsten Krastel for their valuable support in conducting the microbiology procedures of this study.

\section{REFERENCES}

1. Byström A, Sundqvist G. Bacteriologic evaluation of the efficacy of mechanical root canals instrumentation in endodontic treatment. Scand J Dent Res 1981;89:321-8.

2. Sjögren U, Figdor D, Persson S, Sundqvist G. Influence of infection at the time of root filling on the outcome of endodontic treatment of teeth with apical periodontitis. Int Endod J 1997; 30:297-306.

3. Sundqvist G, Figdor D, Persson S, Sjögren U. Microbiologic analysis of teeth with failed endodontic treatment and the outcome of conservative re-treatment. Oral Surg Oral Med Oral Pathol Oral Radiol Endod 1998;85:86-93.

4. Friedman S, Komorowski R, Maillet W, Klimaite R, Nguyen $\mathrm{HQ}$, Torneck CD. In vivo resistance of coronally induced bacterial ingress by an experimental glass ionomer cement root canal sealer. J Endod 2000;26:1-5.

5. Jung S, Safavi K, Spångberg L. The effectiveness of chlorhexidine in the prevention of root canal reinfection [abstract]. J Endod 1999;25:288.

6. Emilson CG. Susceptibility of various microorganisms to chlorhexidine. Scand J Dent Res 1977;85:255-65.

7. Davies A. The mode of action of chlorhexidine. J Periodontal Res Suppl 1973;12:68-75.

8. Delany GM, Patterson SS, Miller CH, Newton CW. The effect of chlorhexidine gluconate irrigation on the root canal flora of freshly extracted necrotic teeth. Oral Surg Oral Med Oral Pathol 1982;53:518-23.

9. Ohara PK, Torabinejad M, Kettering JD. Antibacterial effects of various endodontic irrigants on selected anaerobic bacteria. Endod Dent Traumatol 1993;9:95-100.

10. Vahdaty A, Pitt Ford TR, Wilson RF. Efficacy of chlorhexidine in disinfecting dentinal tubules in vitro. Endod Dent Traumatol 1993;9:243-8.

11. Jeansonne MJ, White RR. A comparison of $2.0 \%$ chlorhexidine gluconate and $5.25 \%$ sodium hypochlorite as antimicrobial endodontic irrigants. J Endod 1994;20:276-8.

12. Siqueira JF, Batista MMD, Fraga RC, De Uzeda M. Antibacterial effects of endodontic irrigants on black-pigmented gram-negative anaerobes and facultative bacteria. J Endod 1998;24:414-6.

13. Barbosa CA, Goncalves RB, Siqueira JF Jr, De Uzeda M. Evaluation of the antibacterial activities of calcium hydroxide, chlorhexidine, and camphorated paramonochlorophenol as intracanal medicament. A clinical and laboratory study. J Endod 1997;23: 297-300.

14. Basson NJ, Tait CM. Effectiveness of three root canal medicaments to eliminate Actinomyces israelii from infected dentinal tubules in vitro. SADJ 2001;56:499-501.

15. Kuruvilla JR, Kamath MP. Antimicrobial activity of $2.5 \%$ sodium hypochlorite and $0.2 \%$ chlorhexidine gluconate separately and combined, as endodontic irrigants. J Endod 1998; 24:472-6.

16. Ayhan H, Sultan N, Cirak M, Ruhi MZ, Bodur H. Antimicrobial effects of various endodontic irrigants on selected microorganisms. Int Endod J 1999;32:99-102.

17. D' Arcangelo C, Varvara G, De Fazio P. An evaluation of the action of different root canal irrigants on facultative aerobicanaerobic, obligate anaerobic, and microaerophilic bacteria. J Endod 1999;25:351-3.

18. Parsons GJ, Patterson SS, Miller CH, Katz S, Kafrawy AH, Newton CW. Uptake and release of chlorhexidine by bovine pulp and dentin specimens and their subsequent acquisition of antibacterial properties. Oral Surg Oral Med Oral Pathol 1980;49: $455-9$

19. Ferretti GA, Brown AT, Raybould TP, Lillich TT. Oral antimi- crobial agentsCchlorhexidine [review]. NCI Monogr 1990;9: 51-5.

20. White RR, Hays GL, Janer LR. Residual antimicrobial activity after canal irrigation with chlorhexidine. J Endod 1997;23:22931.

21. Komorowski R, Grad H, Wu XY, Friedman S. Antimicrobial substantivity of chlorhexidine-treated bovine root dentin. J Endod 2000;26:315-7.

22. Heling I, Sommer M, Steinberg D, Friedman M, Sela MN. Microbiological evaluation of efficacy of chlorhexidine in sustained release device for dentine sterilization. Int Endod J 1992; 25:15-9.

23. Heling I, Steinberg D, Kenig S, Gravilovich I, Sela MN, Friedman M. Efficacy of sustained release device containing chlorhexidine and $\mathrm{Ca}(\mathrm{OH})_{2}$ in preventing secondary infection of dentinal tubules. Int Endod J 1992;25:20-4.

24. Lenet BJ, Komorowski R, Wu XY, Huang J, Grad H, Lawrence $\mathrm{HP}$, et al. Antimicrobial substantivity of bovine root dentin exposed to different chlorhexidine delivery vehicles. J Endod 2000;26:652-5.

25. Ørstavik D, Haapasalo M. Disinfection by endodontic irrigants and dressings of experimentally infected dentinal tubules. Endod Dent Traumatol 1990;6:142-9.

26. Huang J, Wong HL, Zhou Y, Wu XY, Grad H, Komorowski R, et al. In vitro studies and modeling of a controlled-release device for root canal therapy. J Control Release 2000;67:293-307.

27. Tanriverdi F, Esener T, Erganis O, Belli S. An in vitro test model for investigation of disinfection of dentinal tubules infected with Enterococcus faecalis. Braz Dent J 1997;8:67-72.

28. Haapasalo M, Ørstavik D. In vitro infection and disinfection of dentinal tubules. J Dent Res 1987;66:375-9.

29. Stevens RH, Grossman LI. Evaluation of the antimicrobial potential of calcium hydroxide as an intracanal medicament. J Endod 1983;9:372-4.

30. Byström A, Claesson R, Sundqvist G. The antibacterial effect of camphorated paramonochlorophenol, camphorated phenol and calcium hydroxide in treatment of infected roots canals. Endod Dent Traumatol 1985;1:170-5.

31. Dahlen G, Samuelsson W, Molander A, Reit C. Identification and antimicrobial susceptibility of enterococci isolated from the root canal. Oral Microbiol Immunol 2000;15:309-12.

32. Peciuliene V, Balciuniene I, Eriksen HM, Haapasalo M. Isolation of Enterococcus faecalis in previously root-filled canals in a Lithuanian population. J Endod 2000;26:593-5.

33. Hancock HH III, Sigurdsson A, Trope M, Moiseiwitsch J. Bacteria isolated after unsuccessful endodontic treatment in a North American population. Oral Surg Oral Med Oral Pathol Oral Radiol Endod 2001;91:579-86.

34. Waltimo T, Ørstavik D, Siren E, Haapasalo M. In vitro susceptibility of Candida albicans to four disinfectants and their combinations. Int Endod J 1999;32:421-9.

35. Lambrianidis T, Margelos J, Beltes P. Removal efficiency of calcium hydroxide dressing from the root canal. J Endod 1999; 25:85-8.

36. Barthel CR, Zimmer S, West G, Roulet JF. Bacterial leakage in obturated root canals following the use of different intracanal medicaments. Endod Dent Traumatol 2000;16:282-6.

37. Cervone F, Tronstad L, Hammond B. Antimicrobial effect of chlorhexidine in a controlled release delivery system. Endod Dent Traumatol 1990;6:33-6.

Reprint requests:

Bettina Basrani, DDS, PhD

Department of Dental Clinical Sciences

Faculty of Dentistry

Dalhousie University

5981 University Avenue

Halifax, NS B3H 3J5

bettina.basrani@dal.ca 